\title{
Effects of bortezomib in sensitizing human prostate cancer cell lines to NK-mediated cytotoxicity
}

\author{
Wei Hu ${ }^{1}$, Rui-Rui Zheng ${ }^{1}$, Hui-Xia Cui ${ }^{1}$, Dan Yue ${ }^{2}$, Yong Wang ${ }^{3}$ and You-Hong Jiang ${ }^{1}$ \\ The proteasome inhibitor, bortezomib, has been demonstrated to sensitize tumor cells to tumor necrosis factor-related
} apoptosis-inducing ligand (TRAIL)-mediated apoptosis. Natural killer (NK) cells represent potent antitumor effector cells. They also express TRAIL. Therefore, we investigated whether bortezomib could sensitize tumor cells to NK cell-mediated killing, and have the same effect in human prostate cancer cell lines (LNCaP and DU145). We found that bortezomib strongly inhibits proliferation in both cell lines. Furthermore, compared with LNCaP cells, DU145 cells are more sensitive to bortezomib-induced apoptosis. However, bortezomib is unable to sensitize these two cell lines to NK cell-mediated killing in short-term assays. In long-term assays, we found that killing mediated by activated NK cells following bortezomib treatment leads to greater antitumor effects than either treatment alone. In addition, treatment with bortezomib causes these cells to upregulate apoptosis-related mRNA as well as death receptors and downregulate the major histocompatibility class (MHC)-I molecule on the cell surface of DU145 cells. In contrast, LNCaP cells are not sensitized by this treatment. Death receptors and the MHC-I molecule did not change in this cell line. These data suggest that bortezomib can be used to sensitize prostate cancer cells to NK cell-mediated killing and improve current cancer therapies. This therapeutic strategy may be more effective in patients with androgen-insensitive prostate cancer.

Asian Journal of Andrology (2012) 14, 695-702; doi:10.1038/aja.2012.59; published online 20 August 2012

Keywords: bortezomib; DU145 cells; human prostate cancer; LNCaP cells; NK cells

\section{INTRODUCTION}

Prostate cancer is the most common malignant tumor in men and the second leading cause of cancer death in the United States and Europe. In recent years, following the progress of diagnostic techniques, the incidence has increased in Asia. ${ }^{1}$ Most prostate cancer patients encounter tumor recurrence within 1-3 years after treatment. At this stage, prostate cancer loses androgen-dependency and becomes androgen- or hormone-insensitive or refractory and the prognosis is very poor. ${ }^{2}$ Therefore, new treatment modalities are required.

Immunotherapy is a promising treatment modality for malignant tumors. Natural killer (NK) cells are a subset of lymphocytes capable of mediating cytotoxicity against tumor cells and virally infected cells, and thus constitute a key component of the innate immune system. ${ }^{3}$ NK cells lyse tumor targets directly via the perforin/granzyme pathway. They also express Fas ligand and tumor necrosis factor-related apoptosis-inducing ligand (TRAIL), which directly triggers death receptor pathways inducing tumor apoptosis. ${ }^{4}$ Death receptors including Fas, TNFR1, TRAIL-R1/DR4, TRAIL-R2/DR5, DR3 and DR6 share a conserved death domain that is triggered by adaptor molecules which activate executioner caspases and initiate apoptosis. ${ }^{5}$ Recognition of targets by NK cells is regulated through a balance of activated and inhibitory signals. Even in the presence of an activated ligand, inhibitory ligands can initiate overriding signals that culminate in a net suppression of NK-cell function. NK cells recognize major histocompatibility class (MHC) I and class I-like molecules through surface expression of killer immunoglobulin-like receptors (KIRs). MHC I molecules on tumor cells ligate NK cell-inhibitory KIR, and suppress NK-cell function. The inactivation of NK cells by self-human leukocyte antigen (HLA) molecules is a potential mechanism by which malignant cells evade host NK cell-mediated immunity. ${ }^{6}$

Bortezomib, a proteasome inhibitor, has been approved for the treatment of multiple myeloma, and is currently being investigated in other cancers as a single agent or in combination with chemotherapeutic regimens. ${ }^{7}$ It selectively binds to and inhibits the chymotrypticlike activity of the proteasome at nanomolar concentrations. Proteasome inhibition is associated with decreased tumor proliferation and increased apoptosis. ${ }^{8}$ Sayers et al. ${ }^{9}$ previously showed that bortezomib could sensitize tumor cells to TRAIL-mediated lysis, in part due to a reduction in the anti-apoptotic protein cellular FLICE inhibitory protein. TRAIL represents one cytotoxic pathway used by immune effector cells such as T and NK cells to lyse target cells. These immune effector cells have been shown to mediate potent antitumor effects both in vitro and in vivo. ${ }^{10}$

Here, we examine two well-known human prostate cancer cell lines (LNCaP and DU145). LNCaP retains many features of earlystage disease (androgen-sensitive and non-metastatic). In contrast, DU145 is not only locally aggressive but also highly metastatic, and it has a low expression of androgen receptor protein and nonresponsiveness of androgen receptor to androgens in vitro and in

${ }^{1}$ Cancer Research Institute, First Affiliated Hospital, China Medical University, Shenyang 110001, China and ${ }^{2}$ Department of Laboratory Medicine, Tianjin Medical University, Tianjin 300192, China and ${ }^{3}$ Tianjin Institute of Urology, Tianjin Medical University, Tianjin 300192, China 
vivo. ${ }^{11}$ In our present study, we want to study whether bortezomib could sensitize prostate cancer cells to NK cell-mediated killing and its mechanism. This study may supply a more effective therapeutic strategy for androgen-insensitive prostate cancer.

\section{MATERIALS AND METHODS}

\section{Preparation of NK cells}

Human NK cells were isolated by negative depletion of peripheral blood mononuclear cells obtained from healthy volunteers using the NK cell isolation kit ( $\&$ DD, Minneapolis, MN, USA) according to the manufacturer's protocol. Briefly, the Percoll discontinuous density gradient centrifugation method was used to isolate peripheral blood mononuclear cells. Cells at a density of $1 \times 10^{8}$ cells $\mathrm{ml}^{-1}$ were suspended in cold reaction buffer prior to the procedure. Cells $\left(1 \times 10^{8}\right.$ cells, $0.5-1.0 \mathrm{ml}$ volume) were transferred into a 5-ml tube. A $25-\mu \mathrm{l}$ volume of biotinylated antibody was added. The cell-antibody suspension was gently mixed, avoiding bubble formation, and incubated at $4{ }^{\circ} \mathrm{C}$ in a refrigerator for $15 \mathrm{~min}$. Fifty microliters of MagCellect beads were added to the cell suspension, mixed gently and incubated at $4{ }^{\circ} \mathrm{C}$ in a refrigerator for $15 \mathrm{~min}$. At the end of the incubation period, the cell suspension was transferred to a $50-\mathrm{ml}$ conical centrifuge tube, and the cells were washed with 15 volumes of reaction buffer and centrifuged at $300 \mathrm{~g}$ for $8 \mathrm{~min}$. The supernatant was completely pipetted out, and the cell pellet was resuspended in $2 \mathrm{ml}$ of reaction buffer and transferred to a new 5-ml tube. The tube was placed in a MagCellect Magnet that had been positioned horizontally to accommodate 5-ml tubes and incubated for $6 \mathrm{~min}$ at room temperature. Magnetically tagged cells will migrate toward the magnet, leaving the unwanted cells in suspension. While the tube was in the magnet, the reaction supernatant was carefully aspirated using a pipette and discarded. The tube containing the desired magnetically trapped cells was removed from the magnet and the cells were resuspended in $2 \mathrm{ml}$ of reaction buffer by gently pipetting up and down. This step was repeated. The resultant cell fraction contained the NK cells. The NK cells were cultured in RPMI 1640 medium (GIBCO BRL, Gaithersburg, MD, USA) supplemented with $10 \%$ fetal calf serum (FCS; HyClone, Logan, UT, USA), and $500 \mathrm{U} \mathrm{ml}^{-1}$ recombinant human IL-2 (Roche, Nutley, NJ, USA) for $24 \mathrm{~h}$ to activate the NK

a

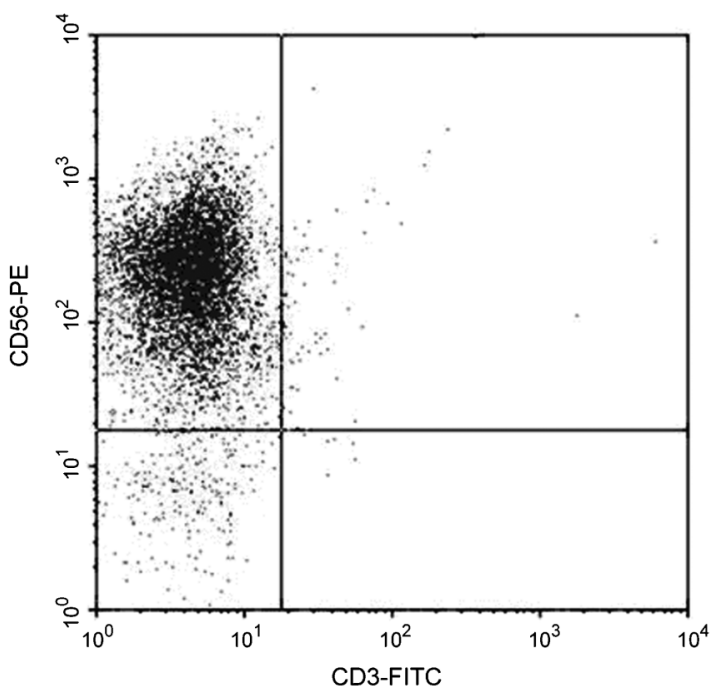

cells. The purity of human NK cells was typically greater than $85 \%$ as determined by staining for CD3, CD16 and CD56 (Biolegend, San Diego, CA, USA), respectively (Figure 1). Written informed consent was obtained from each healthy volunteer in accordance with the Declaration of Helsinki for the use of cells from leukapheresis products for research, according to the requirements of the Institutional Review Board of the National Heart, Lung, and Blood Institute (Bethesda, MD, USA).

\section{Cell culture}

The LNCaP and DU145 prostate cancer cell lines were obtained from American Type Culture Collection (Rockville, MD, USA). The LNCaP cells were maintained in F12 medium (GIBCO BRL), and the DU145 cells were cultured in RPMI 1640 medium (GIBCO BRL). All cell lines were supplemented with $10 \%(\mathrm{v} / \mathrm{v})$ FCS (HyClone) and incubated at $37{ }^{\circ} \mathrm{C}$ with $5 \% \mathrm{CO}_{2}$.

\section{Reagents}

Bortezomib (PS-341; Velcade) was provided by Millennium Pharmaceuticals (Cambridge, MA, USA). Stock solutions of bortezomib were made at $1 \mathrm{mg} \mathrm{ml}^{-1}$ in phosphate-buffered saline and frozen at $-80{ }^{\circ} \mathrm{C}$. The stock solutions were thawed and diluted to $1 \mu \mathrm{g} \mathrm{ml}^{-1}$ with RF-10 complete medium and used within 2 weeks of dilution. Bortezomib solutions were protected from light at all times.

\section{Flow cytometry}

The LNCaP and DU145 cells were seeded onto six-well plates at a density of $2.0 \times 10^{5}$ cells per $2 \mathrm{ml}$ complete medium and allowed to attach for $24 \mathrm{~h}$. Following this incubation, the cells were washed once with serum-free F12 or RPMI 1640 and replaced with a medium containing $10 \% \mathrm{FCS}$ and $0 \mathrm{nmol} 1^{-1}$ or $20 \mathrm{nmol}^{-1}$ bortezomib and incubated for $24 \mathrm{~h}$. Cells were then digested using trypsogen. Approximately $2 \times 10^{5} \mathrm{LNCaP}$ and DU145 cells per sample were brought to a single-cell suspension and washed with blocking buffer ( $1 \%$ fetal bovine serum and $1 \%$ human antibody serum in phosphatebuffered saline). Cells were labeled with phycoerythrin (PE)-antiDR5 (DJR2-4), PE-anti-Fas (DX2) and PE-anti-HLA-A, B, C (W6/ 32) (Biolegend, San Diego, CA, USA). Cellular labeling was performed

b

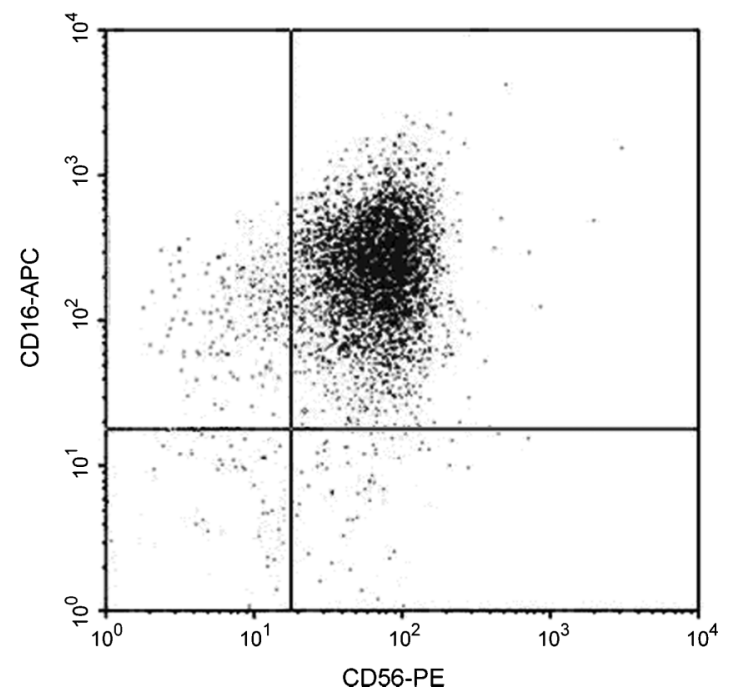

Figure 1 The purity of human NK cells was determined by staining for CD3, CD16 and CD56: (a) the ratio of $\mathrm{CD}^{-} \mathrm{CD}^{-} 6^{+}$cells; (b) the ratio of $\mathrm{CD} 16^{+} \mathrm{CD} 56^{+}$cells. NK, natural killer. 
at $4{ }^{\circ} \mathrm{C}$ for $30 \mathrm{~min}$. All samples were analyzed on a Becton Dickinson fluorescence activated cell sorter (FACScan) flow cytometer using Cell-Quest software (BD Biosciences, San Jose, CA, USA).

\section{Proliferation and killing assay}

The LNCaP and DU145 cells were suspended in F12 or RPMI 1640 medium with $10 \%$ FCS at a concentration of $1 \times 10^{4}$ cells $\mathrm{ml}^{-1}$. The cell suspension in $100 \mu \mathrm{l}$ was added to 96-well flat-bottomed culture plates and incubated at $37{ }^{\circ} \mathrm{C}$, in $5 \% \mathrm{CO}_{2}$ for $24 \mathrm{~h}$. After the cells adhered, bortezomib was added to the wells at the following concentrations: $0,5,10,15,20$ and $25 \mathrm{nmol}^{-1}$. F12 or RPMI 1640 medium with $10 \%$ FCS was used as a negative control and incubated at $37^{\circ} \mathrm{C}$, in $5 \% \mathrm{CO}_{2}$ for 24,48 or $72 \mathrm{~h}$. For the killing assay, 10 or $20 \mathrm{nmol} \mathrm{l}^{-1}$ bortezomib was incubated for $24 \mathrm{~h}$. After incubation, $10 \mu \mathrm{lCCK}-8 \mathrm{kit}$ (Dodindo Laboratories, Kumamoto, Japan) was added to each well, oscillated for $1 \mathrm{~min}$ on an oscillator and incubated at $37^{\circ} \mathrm{C}$ and in $5 \%$ $\mathrm{CO}_{2}$ for 1.5 h continuously. After incubation, absorbances were read on a Dynatech MR 5000 plate reader at $490 \mathrm{~nm}$. This experiment was repeated five times.

\section{Apoptosis detection kit (annexin-V binding)}

This assay was performed according to the manufacturer's instructions. LNCaP and DU145 cells were seeded onto six-well plates and incubated for $24 \mathrm{~h}$, and then treated with $0,5,10,15,20$ and $25 \mathrm{nmol} \mathrm{l}^{-1}$ bortezomib. After $24 \mathrm{~h}$, the cells were trypsinized, counted and sedimented, then washed with cold phosphate-buffered saline and resuspended in $500 \mu \mathrm{l}$ binding buffer. FITC-conjugated annexin-V $(10 \mu \mathrm{l})$ and propidium iodide $(10 \mu \mathrm{l})$ (Keygen, Nanjing, China) were added to each sample, and the mixture was incubated at $4{ }^{\circ} \mathrm{C}$ in the dark for 5 min. The cells were then immediately subjected to FACS analysis (BD FAC-S Calibur, San Jose, CA, USA).

\section{Quantitative real-time PCR}

Total RNA was isolated from cells using Trizol reagent (Invitrogen, Carlsbad, CA, USA). RNA (500 ng) was then reverse transcribed into complementary DNA using oligo(dT) primers and AMV Reverse Transcriptase (Takara, Shiga, Japan). Complementary DNA was synthesized by incubating the reaction mix at $25^{\circ} \mathrm{C}$ for $5 \mathrm{~min}, 42{ }^{\circ} \mathrm{C}$ for $30 \mathrm{~min}$ and $85^{\circ} \mathrm{C}$ for $5 \mathrm{~min}$. cDNA samples were stored at $-20^{\circ} \mathrm{C}$ until use.

Quantitative real-time PCR was performed using the MyiQ realtime PCR detection system (Bio-Rad, Irvine, CA, USA). PCR was carried out in a $50-\mu \mathrm{l}$ reaction volume: $25 \mu \mathrm{l}$ of iQ SYBR Green Supermix, $0.5 \mu$ l each of specific primer mix $\left(100 \mathrm{nmol}^{-1}\right.$ as the final

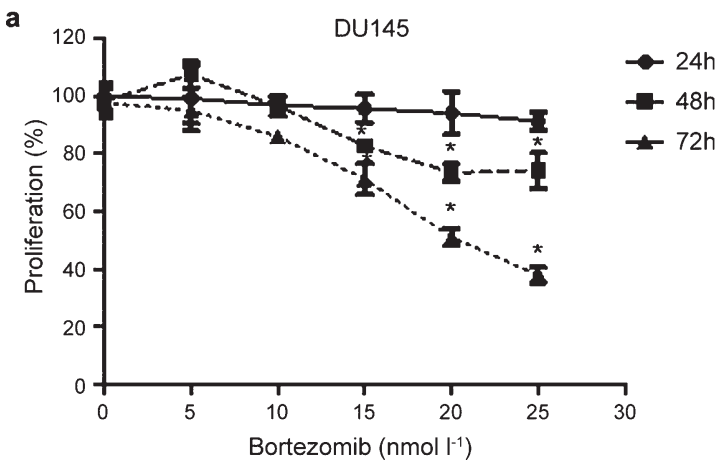

concentration of each primer), $2 \mu \mathrm{l}$ of complementary DNA and $19 \mu \mathrm{l}$ of nuclease-free water. Samples were run in duplicate in 96-well plates (Bio-Rad). The primers used for quantitative real-time PCR were as follows:

DR5: forward 5'-AGCTAAGTCCCTGCACCACGA 3', reverse 5'-TGTACAATCACCGACCTTGACCA-3';

Fas: forward 5'-CAACAACCATGCTGGGCATC-3', reverse 5' TGATGTCAGTCACTTGGGCATTAAC 3';

HLA-C: forward 5'-TCCTGGTTGTCCTAGCTGTC-3', reverse 5'-CAGGCTTTACAAGTGATGAG-3';

GAPDH: forward 5'-GAAGGTGAAGGTCGGAGTC-3', reverse 5'-GAAGATGGTGATGGGATTTC-3'

The PCR cycles consisted of denaturation at $95{ }^{\circ} \mathrm{C}$ for $15 \mathrm{~s}$, and annealing as well as extension at $60^{\circ} \mathrm{C}$ for $1 \mathrm{~min}$ for $40-60$ cycles. Each sample was analyzed twice. The threshold cycle $(\mathrm{Ct})$ was defined as the fractional cycle number at which the fluorescence exceeds the given threshold. The obtained data were translated into the $\log 2$ scale. The $2^{-\Delta \Delta \mathrm{Ct}}$ method was used to analyze the relative expression of the target gene.

\section{Statistical analysis}

Statistical analysis was performed using GraphPad Prism 4 software (GraphPad, San Diego, CA, USA). One-way and two-way analysis of variance were used where appropriate.

\section{RESULTS}

Bortezomib inhibits viability and proliferation on both DU145 and LNCaP cells

We sought to determine if the DU145 and LNCaP cell lines would display sensitization to bortezomib in terms of viability and proliferation. The effects of bortezomib on DU145 and LNCaP cells are shown in Figure 2. The proliferation of tumor cells was determined by CCK-8 after DU145 and LNCaP cells had been exposed to varying concentrations of bortezomib for 24,48 or $72 \mathrm{~h}$. Cells incubated with 15,20 or $25 \mathrm{nmol} 1^{-1}$ bortezomib displayed a significant decrease $(P<0.01)$ in proliferation after $48 \mathrm{~h}$ (Figure 2).

\section{Bortezomib induces apoptosis in both DU145 and LNCaP cells} DU145 and LNCaP cells were exposed to varying concentrations of bortezomib for $24 \mathrm{~h}$, and then apoptosis was analyzed using annexin- $\mathrm{V}$ and propidium iodide. DU145 cells displayed a slight, but significant $(P<0.01)$ increase in apoptosis at or above $15 \mathrm{nmoll}^{-1}$ of bortezomib,

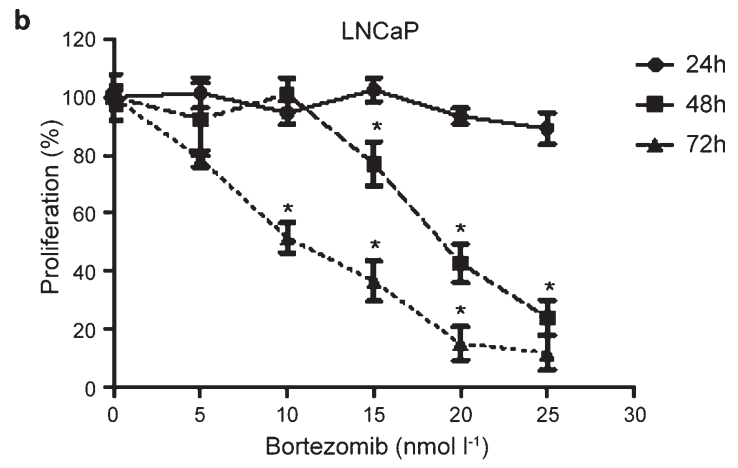

Figure 2 Bortezomib treatment in vitro causes a significant decrease in proliferation in prostate cancer cells. DU145 (a) or LNCaP (b) cells were exposed to 0, 5, 10, 15,20 or $25 \mathrm{nmol} \mathrm{I}^{-1}$ bortezomib. After 24,48 and $72 \mathrm{~h}$, the proliferation of tumor cells was determined by CCK-8 assay. Data were determined by two-way ANOVA with Bonferroni post-test. ( ${ }^{*} P<0.01$, compared with $0 \mathrm{nmol} \mathrm{I}^{-1}$ bortezomib group). ANOVA, analysis of variance. 
whereas higher concentrations were required to stimulate significant apoptosis in LNCaP cells (Figure 3).

Short-term killing assays do not reflect an increase in the sensitization of prostate cancer cells by NK cell-mediated killing We sought to determine if bortezomib could sensitize tumor cells to human NK cell-mediated killing in vitro. DU145 and LNCaP cells were incubated with varying concentrations of bortezomib for $48 \mathrm{~h}$. The highest dose of $20 \mathrm{nmol}^{-1}$ was chosen because higher concentrations displayed cytotoxic effects in both cell lines, while the $20 \mathrm{nmol} \mathrm{l}^{-1}$ dose demonstrated growth inhibition, but minimal cytotoxic effects (Figures 2 and 3). The treated DU145 and LNCaP cells were cocultured with $1: 1,4: 1,16: 1$ and $32: 1$ effector-to-target (E:T) ratios for $24 \mathrm{~h}$, and then washed and assayed using CCK-8. Only a slight increase in NK-mediated lysis was observed in these short-term assays (Figure 4). We hypothesized that in order to see the effects of other modes of NK cell cytotoxicity, a long-term assay would be needed.

Bortezomib increases mRNA levels of DR5 and Fas and decreases the expression of HLA-C in DU145 cells, but not in LNCaP cells Previous studies have demonstrated that bortezomib increases the surface expression of death receptors on mouse leukemia cells. ${ }^{12}$ We sought to determine if bortezomib treatment would increase the amount of DR5 and Fas genes in the human prostate cancer line. DU145 and LNCaP cells were exposed to varying amounts of bortezomib for $24 \mathrm{~h}$ and then mRNA levels were determined by real-time
PCR analysis. With incremental bortezomib concentrations, DU145 cells displayed an insignificant but marked increase in the levels of DR5 and Fas $(P<0.01)$. However, these genes showed very small changes in LNCaP cells. The inactivation of NK cells by self-HLA molecules is a potential mechanism by which malignant cells evade host NK cellmediated immunity, particularly HLA-C and Bw4 molecules. ${ }^{13}$ We detected the expression of HLA-C in these two cell lines. We found that, the expression of HLA-C was decreased after treatment with bortezomib in DU145 cells $(P<0.01)$, but not in LNCaP cells (Figure 5).

Bortezomib treatment increases the surface expression of DR5 and Fas and decreases the expression of HLA-A, B, C in DU145 cells, but not in LNCaP cells

To determine if the increase in death receptor mRNA correlated with an increase in cell surface expression, we used flow cytometry to determine the surface levels of DR5, Fas and HLA-A, B, C with or without bortezomib treatment. DU145 and LNCaP cells were maintained in normal culture conditions or treated with $20 \mathrm{nmol} \mathrm{l}^{-1}$ bortezomib for $24 \mathrm{~h}$ before labeling with PE-conjugated anti-DR5, antiFas and anti-HLA-A, B, C. In DU145 cells, the surface expression of both DR5 and Fas was upregulated in cells which received bortezomib treatment, and the expression of HLA-A, B, C was decreased $(P<0.01)$. In contrast, all three proteins in LNCaP cells were not expressed (Figure 6). These data correspond with the death receptor mRNA expression displayed in Figure 5.
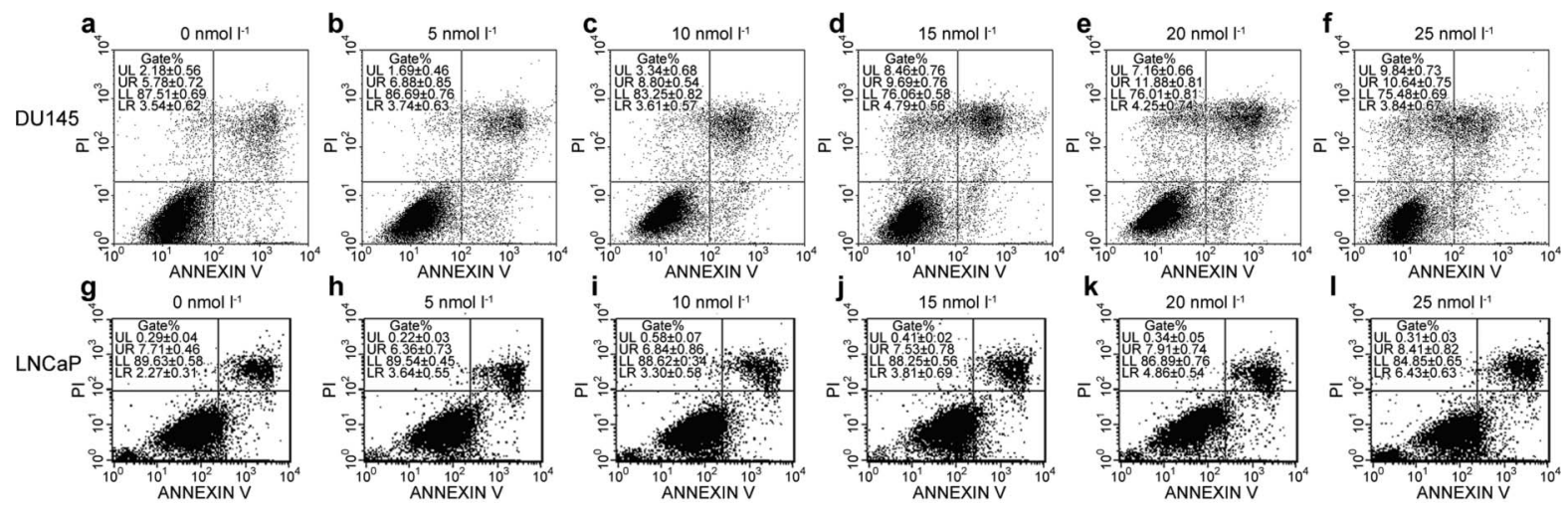

m

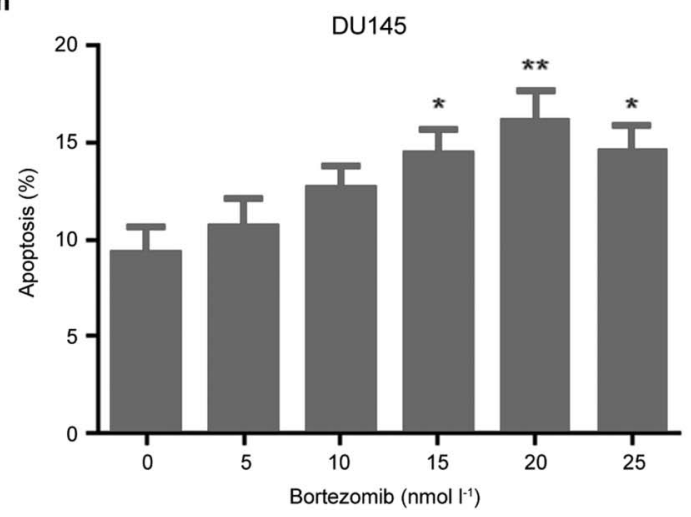

n

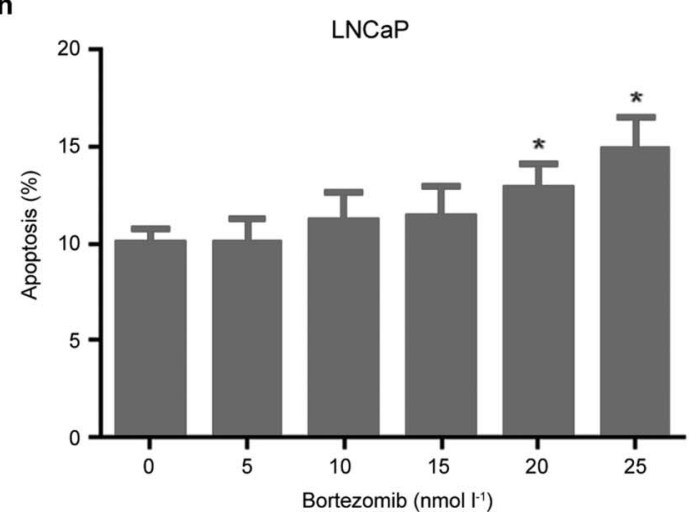

Figure 3 Bortezomib treatment in vitro causes a slight but significant increase in apoptosis in prostate cancer cells. DU145 or LNCaP cells were incubated with varying concentrations of bortezomib for $24 \mathrm{~h}$, and then stained with annexin-V and propidium iodide to determine the percentage of apoptotic cells. (a) - (f) and ( $\mathbf{m})$ Apoptosis ratio of DU145 cells. (g)-(I) and (n) Apoptosis ratio of LNCaP cells. Data were determined by one-way ANOVA with Dunnett's post-test. (*P<0.01, **P<0.001, compared with $0 \mathrm{nmol} \mathrm{I}^{-1}$ bortezomib group). ANOVA, analysis of variance. 


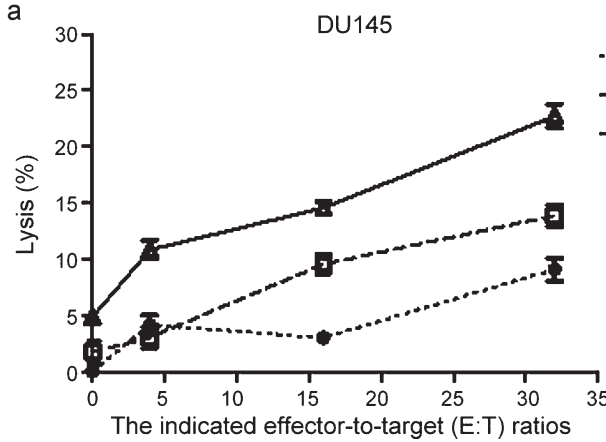

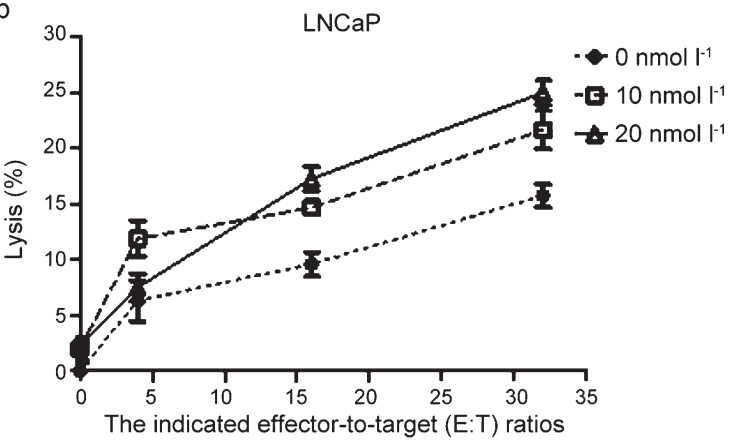

Figure 4 Sensitization of prostate cancer cells to NK cell killing is not shown in short-term assays. DU145 (a) or LNCaP (b) cells were placed in a culture flask and treated with bortezomib at either 10 or $20 \mathrm{nmol} \mathrm{I}{ }^{-1}$ or left untreated for $48 \mathrm{~h}$. The prostate cancer cells were then washed twice and resuspended in media. The activated NK effector cells were added at the indicated $\mathrm{E}: \mathrm{T}$ ratios, with a maximum $\mathrm{E}: \mathrm{T}$ of $32: 1$. The CCK-8 assay was then used to evaluate killing of the target cells. No significant difference was found between the treatments, as determined by two-way ANOVA with a Bonferroni post-test. ANOVA, analysis of variance.; E: T, effector-to-target; NK, natural killer.

\section{Bortezomib-treated prostate cancer cells are sensitized to NK killing in the long-term assays}

Only a slight increase in NK cell killing of prostate cancer cells after bortezomib sensitization was observed in the short-term cytotoxicity assays (Figure 4). Therefore, we hypothesized that bortezomib sensitization would increase markedly if we used a longer-term assay. To address this, we treated DU145 and LNCaP cells with or without $20 \mathrm{nmol}^{-1}$ bortezomib for $48 \mathrm{~h}$. The cells were then counted, equal numbers were plated and the cells were allowed to adhere to plates for $24 \mathrm{~h}$. NK cells were activated by adding $500 \mathrm{U} \mathrm{ml}^{-1}$ recombinant human IL-2 for $24 \mathrm{~h}$. Tumor cells were washed and incubated with activated NK cells at an E: T ratio of $1: 1$ for 6 days. All cells were then removed from the plates and apoptosis was analyzed using annexin- $\mathrm{V}$ and propidium iodide. The 6-day period in which the tumor cells were exposed to NK cells allowed killing through multiple mechanisms to occur, including the ligation of death receptors. ${ }^{12}$ Bortezomib pretreatment resulted in a decreased number of live tumor cells and increased apoptosis after the 6-day period in both DU145 and LNCaP cells $(P<0.01)$. The effects of this treatment were more pronounced in DU145 cells compared with LNCaP cells $(P<0.001)$. NK cells effectively increased the rate of apoptosis in cells in all groups. However, the combination of NK cells and bortezomib pre-treatment resulted in a decrease in proliferation that was lower than each treatment alone (Figure 7).

\section{DISCUSSION}

Molecular targeting in cancer treatment offers great promise in the selective attack of tumor cells. In particular, the augmentation of immune attack will be an effective therapy which could selectively kill rebirth tumor cells in the future. ${ }^{14}$ Transient proteasome inhibition may provide a manageable therapeutic 'window' for the use of proteasome inhibitors in cancer therapy. Other investigators have demonstrated that bortezomib can augment NK cell-mediated killing of mouse leukemia cells. ${ }^{12}$ In this report, we demonstrate that bortezomib
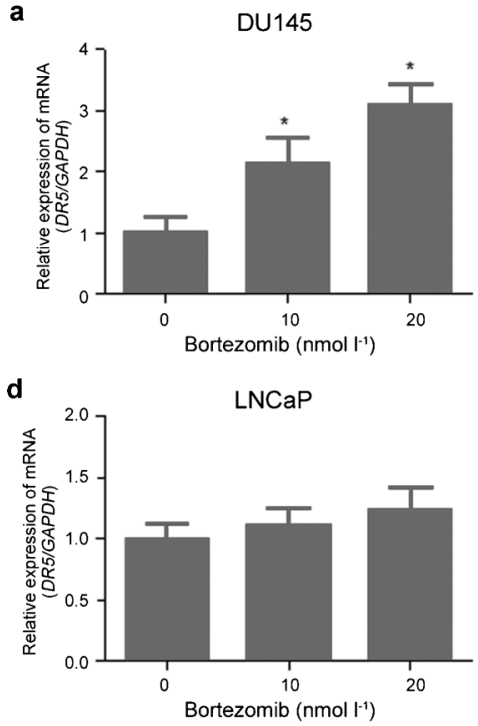

b

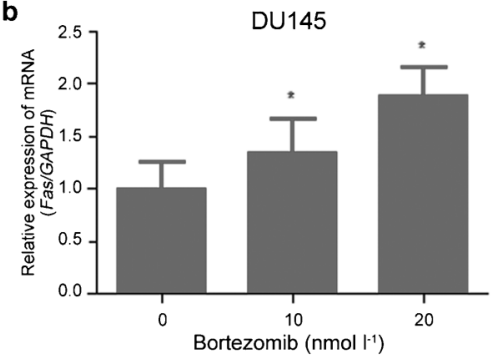

e

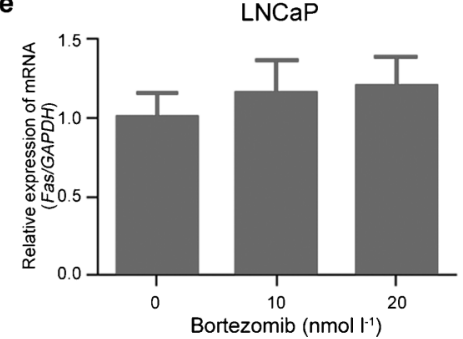

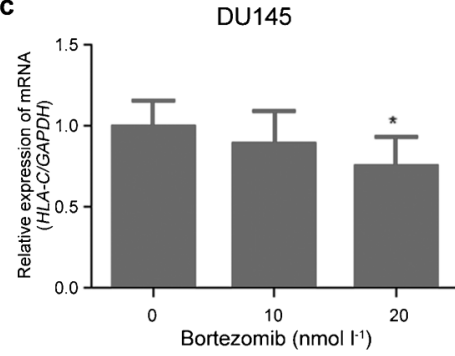

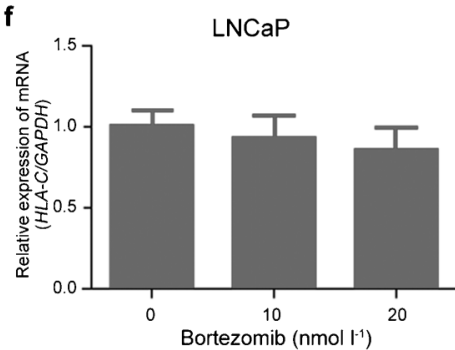

Figure 5 Bortezomib pre-treatment changed the mRNA of proteins associated with apoptosis in DU145 and LNCaP cells. These cells were incubated with 0 , 10 or $20 \mathrm{nmol} \mathrm{I-1}$ of bortezomib for $24 \mathrm{~h}$. RNA was then isolated and real-time PCR analysis was performed to determine the amount of mRNA present in apoptosis-related proteins. (a)-(c) The fold changes in DR5, Fas and HLA-C in DU145 cells, respectively. (d)-(f) The fold changes in DR5, Fas and HLA-C in LNCaP cells, respectively. Although all genes examined displayed an increase, only the increase in the indicated panels proved to be significant. Data were determined by one-way ANOVA with Bonferroni post-test. ( ${ }^{*}<0.01$, compared with 0 nmol $\mathrm{I}^{-1}$ bortezomib group). ANOVA, analysis of variance.; DR5, death receptor 5 ; HLA, human leukocyte antigen. 

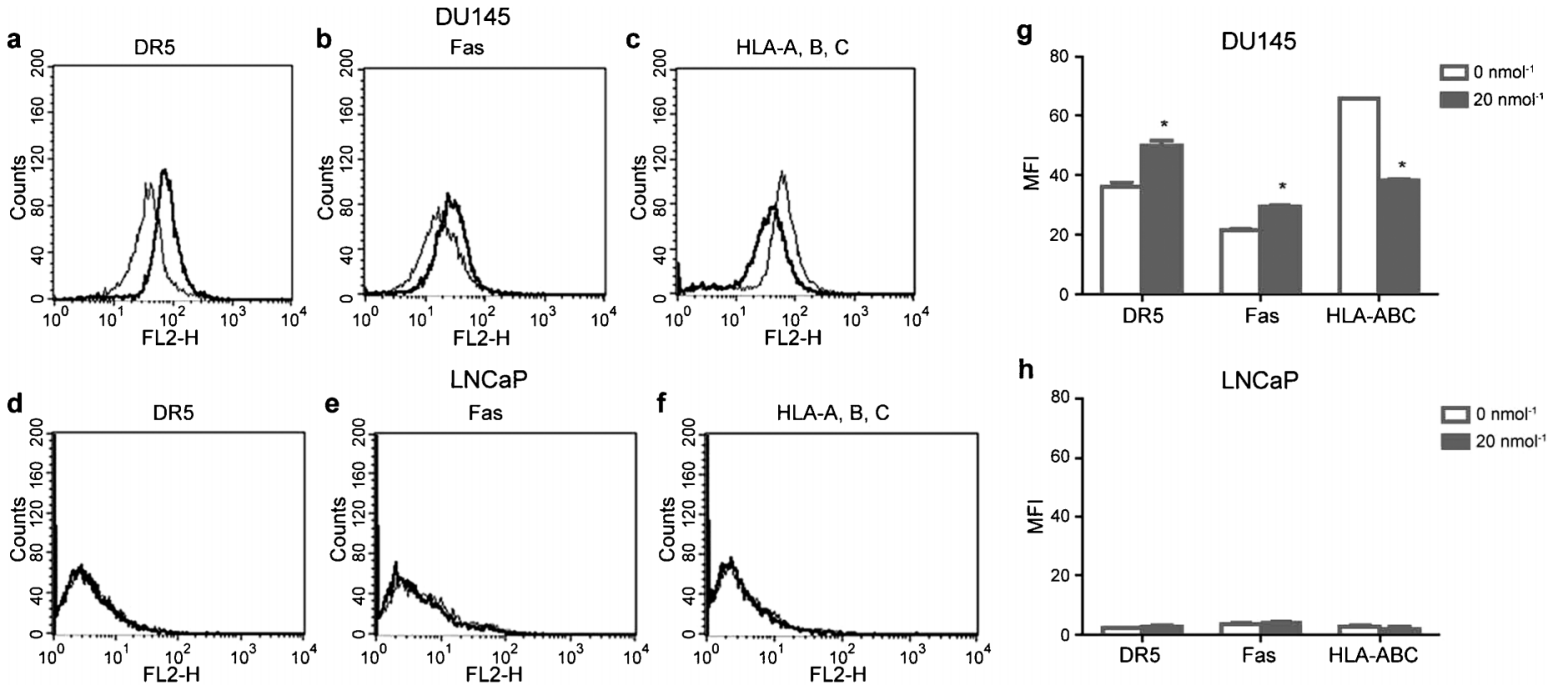

Figure 6 Bortezomib pre-treatment increases the surface expression of DR5 and Fas, and decreases the expression of HLA molecules. DU145 and LNCaP cells were treated with $20 \mathrm{nmol} \mathrm{I}^{-1}$ bortezomib (thick black line) or left untreated (thin black line) for $24 \mathrm{~h}$. Cells were stained with fluorescent-conjugated anti-DR5, anti-Fas and anti-HLA-A, B, C and analyzed by flow cytometry. The MFI was calculated in order to determine the relative amount of the indicated surface protein. (a)-(c) and (g) Mean fluorescence intensity of DU145 cells. (d)-(f) and (h) Mean fluorescence intensity of LNCaP cells. Data were calculated by a two-tailed $t$-test. $(* P<0.01$, compared with $0 \mathrm{nmol} \mathrm{I}^{-1}$ bortezomib group). DR5, death receptor 5; HLA, human leukocyte antigen; MFI, mean fluorescence intensity.
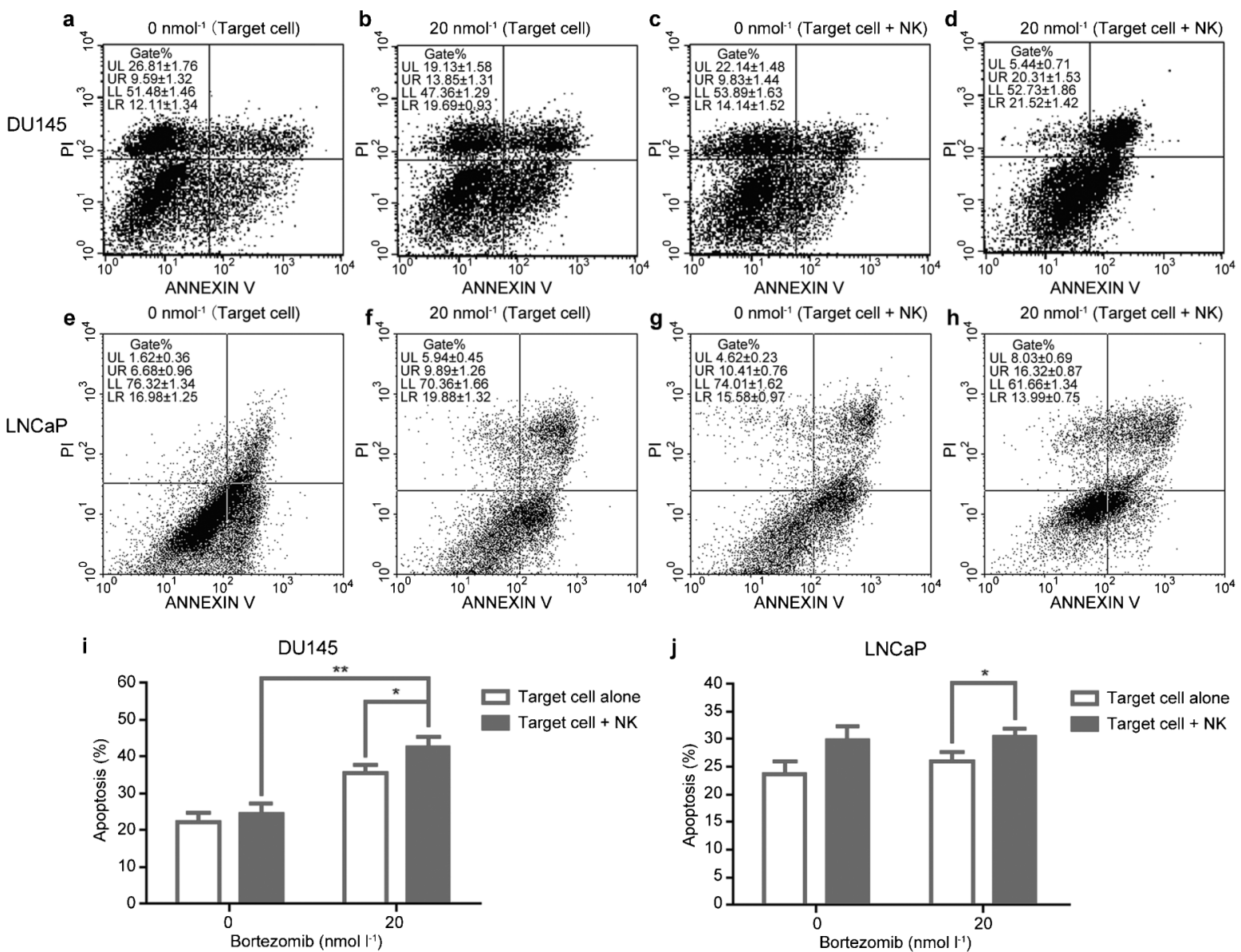

Figure 7 Long-term assays displayed a synergistic increase in NK-mediated killing of DU145 and LNCaP cells after bortezomib pre-treatment; DU145 and LNCaP cells were incubated with $20 \mathrm{nmol} \mathrm{I}^{-1}$ bortezomib or left untreated for $48 \mathrm{~h}$. Target cells were then washed, and allowed to adhere to the wells for $24 \mathrm{~h}$. In some wells, activated NK cells were then added at a $1: 1$ effector-to-target ratio. Plates were incubated for an additional 6 days and then analyzed by flow cytometry using annexin-V and propidium iodide to determine the percentage of apoptotic cells. (a)-(d) and (i) Apoptosis ratio of DU145 cells. (e)-(h) and (j) Apoptosis ratio of LNCaP cells. Data were calculated by a two-tailed $t$-test. ( $* P<0.01,{ }^{* *} P<0.001$, compared with $0 \mathrm{nmol} \mathrm{I}^{-1}$ bortezomib $+\mathrm{NK}$ or $20 \mathrm{nmol} \mathrm{I}^{-1}$ bortezomib group). NK, natural killer. 
increases the antitumor responses mediated by freshly isolated human NK cells in androgen-sensitive and -insensitive prostate cancer cells. The results of our viability and proliferation experiments demonstrate that bortezomib induces strong growth arrest at concentrations greater than $15 \mathrm{nmol} \mathrm{l}^{-1}$ in vitro. In DU145 cells, clinically relevant concentrations of bortezomib induced characteristic apoptosis. On the other hand, a higher concentration of bortezomib was required to stimulate apoptosis in LNCaP cells. This may be because LNCaP cells express wild-type $p 53$ and $p 21$ and high expression of these two genes could inhibit apoptosis. ${ }^{15,16} \mathrm{We}$ also found that bortezomib was unable to efficiently sensitize these two cell lines to NK cell-mediated killing in short-term assays. In long-term assays, we found that killing by activated NK cells following bortezomib treatment led to greater antitumor effects than either treatment alone. In addition, treatment of DU145 cells with bortezomib led to a dose-dependent increase in the transcription of apoptosis-related genes and a decrease in MHC-I molecule genes. These data also correlated with an increase in the surface expression of death ligand receptors DR5 and Fas and downregulation of the MHC-I molecule. In contrast, LNCaP cells were not sensitized by this treatment. The death receptors or MHC-I molecule did not change in this cell line.

Killing via cytotoxic granules has been shown to be the primary mechanism by which NK cells kill in short-term in vitro assays, ${ }^{17}$ although these short-term assays do not reflect the engagement of death ligands on tumor targets. Therefore, it may be more informative to use longer-term assays in which NK cells can use their full complement of killing pathways; these conditions may be a better predictor of how effective the NK cells function. We demonstrate that bortezomib sensitized DU145 and LNCaP cells to killing by activated NK cells. The killing of bortezomib-treated targets in short-term killing assays was not augmented, and enhancement was only observed in long-term tumor outgrowth studies. Furthermore, sensitization was more obvious in DU145 cells.

The precise molecular mechanism by which proteasome inhibition acts to elicit its anticancer apoptotic activity has been difficult to resolve and is likely to be multifactorial. This is largely due to the vast number of cellular pathways regulated by the $26 \mathrm{~S}$ proteasome. We found that bortezomib-pre-treated DU145 and LNCaP cells showed increased surface expression of death receptors DR5 and Fas which correlated with an increase in the transcription apoptotic genes, whereas in LNCaP cells, we did not observe these changes. NK cells possess TRAIL and Fas ligand (FasL), both of which can trigger apoptosis in target cells. These combined effects may present a mechanism by which bortezomib increases the susceptibility of tumor cells to NK cell-mediated killing. This, in part, may explain why bortezomib induced a higher level of apoptosis in DU145 cells. We also observed that activated NK cells slightly increased the percentage of apoptosis in LNCaP cells pre-treated with/without bortezomib after 6 days of coculturing. It is possible that bortezomib can also augment the cytokines produced by NK cells, notably interferon- $\gamma$ and tumor necrosis factor, which may be capable of engaging other death pathways, especially at lower E: T ratios. ${ }^{4}$ However, the increase in death receptors and other components of extrinsic apoptosis does not explain the apparent increase in intrinsic apoptosis. Changes in intrinsic proapoptotic proteins (PUMA, Bax, and so on) or anti-apoptotic proteins (Bcl-2, Bcl-xL, and so on) are probably responsible.

Haplo-identical donor NK cells have been transfused in combination with an autograft in patients with multiple myeloma or after immunosuppression for acute leukemia or renal cell carcinoma. ${ }^{18}$ These studies have demonstrated the activity of haplo-identical KIR-L-mismatched
NK cells; however, therapeutic efficacy has been hampered as only a sub-population of the transferred NK cells was found to be allo-reactive. The bortezomib-induced downregulation of HLA class I and enhanced NK cell-mediated killing indicate that bortezomib can recruit nonalloreactive NK-cell populations. These effects suggest that bortezomib may play an important role in potentiating the antitumor activity of allogeneic KIR-L-mismatched NK cells.

Under normal circumstances, prostate cancer cells display selfHLA molecules, which inactivate autologous NK cells. This also helps to explain the relative lack of activity of adoptively transfused autologous NK cells in the solid tumor setting. In our study, we observed that treatment of DU145 cells with bortezomib led to a decrease in the surface expression of the HLA-ABC protein and HLA-C gene. Owing to cancer cells showing less MHC-I than normal cells, in long-term tumor outgrowth studies, we should see more apoptotic cells die in target coculture with NK cells compared with target cells alone. This demonstrated that bortezomib can overcome KIR-L inhibition of NK cells by prostate cancer cells and make adoptive NK-cell therapy more effective and widen its application. These changes were not observed in LNCaP cells. It is possible that bortezomib kills LNCaP cells in some other way. However, some investigators have observed that human NK cells were highly sensitive to bortezomib in vitro. ${ }^{12}$ The half-life of bortezomib is short, and serum levels present $48 \mathrm{~h}$ after a dose of bortezomib did not adversely affect NK-cell function. However, its function in vivo remains unclear. More studies to identify whether bortezomib could sensitize prostate cancer to NK cellmediated killing in vivo, and tests to ascertain its safety, feasibility and efficacy could be future directions.

Recent progress defines molecular alterations and the contributions to prostate cancer progression and metastasis; however, there is a general consensus that current therapeutic strategies are relatively inactive in patients with androgen-insensitive cancer. The results of this study and others demonstrate that bortezomib can enhance the antitumor ability of NK cells. This effect can be utilized to sensitize solid tumors to NK cell-mediated killing and improve current cancer therapies. This therapeutic strategy may be more efficient in patients with androgen-insensitive prostate cancer.

\section{AUTHOR CONTRIBUTIONS}

WH, RRZ and HXC carried out the study, analyzed the data and wrote the manuscript. DY, YW and YHJ were involved in the study design and data management of the study.

\section{COMPETING FINANCIAL INTERESTS}

The authors declare no competing financial interests.

\section{ACKNOWLEDGMENTS}

This study was supported by the Peak Medical Research Construction Projects of Liaoning Province (No.[2010]017).

1 Zhang L, Yang BX, Zhang HT, Wang JG, Wang HL et al. Prostate cancer: an emerging threat to the health of aging men in Asia. Asian J Androl 2011; 13: 574-8.

2 Kent EC, Hussain $\mathrm{MH}$. The patient with hormone-refractory prostate cancer: determining who, when, and how to treat. Urology 2003; 62: 134-40.

3 Herberman RB, Ortaldo JR. Natural killer cells: their roles in defenses against disease. Science 1981; 214: 24-30.

4 Hallett WH, Murphy WJ. Positive and negative regulation of natural killer cells: therapeutic implications. Semin Cancer Biol 2006; 16: 367-82.

5 Wajant H. Death receptors. Essays Biochem 2003; 39: 53-71.

6 Lanier LL. NK cell receptors. Annu Rev Immunol 1998; 16: 359-93. 
7 Mateos MV, Hernández JM, Hernández MT, Gutiérrez NC, Palomera L et al. Bortezomib plus melphalan and prednisone in elderly untreated patients with multiple myeloma: results of a multicenter phase $1 / 2$ study. Blood 2006; 108: 2165-72.

8 Voorhees PM, Orlowski RZ. The proteasome and proteasome inhibitors in cancer therapy. Annu Rev Pharmacol Toxicol 2006; 46: 189-213.

9 Sayers TJ, Brooks AD, Koh CY, Ma W, Seki N et al. The proteasome inhibitor PS-341 sensitizes neoplastic cells to TRAIL-mediated apoptosis by reducing levels of C-FLIP. Blood 2003; 102: 303-10.

10 Klebanoff, CA, Gattinoni L, Restifo NP. CD8 ${ }^{+}$T-cell memory in tumor immunology and immunotherapy. Immunol Rev 2006; 211: 214-24.

11 Alimirah F, Chen J, Basrawala Z, Xin H, Choubey D. DU-145 and PC-3 human prostate cancer cell lines express androgen receptor: implications for the androgen receptor functions and regulation. FEBS Lett 2006; 17: 2294-300.

12 Hallett WH, Ames E, Motarjemi M, Barao I, Shanker A et al. Sensitization of tumor cells to NK cell-mediated killing by proteasome inhibition. Immunol 2008; 180: 163-70.
13 Leung W, lyengar R, Turner V, Lang P, Bader P et al. Determinants of antileukemia effects of allogeneic NK cells. Immunol 2004; 172: 644-50.

14 Marsoni S, Damia G. Molecular targeting: new therapeuticstrategies to improve tumour apoptosis. Ann Oncol 2004; 15: 229-31.

15 Lashinger LM, Zhu K, Williams SA, Shrader M, Dinney CP et al. Bortezomib abolishes tumor necrosis factor-related apoptosis-inducing ligand resistance via a p21dependent mechanism in human bladder and prostate cancer cells. Cancer Res 2005; 65: 4902-8.

16 Pettaway CA, Pathak S, Greene G, Ramirez E, Wilson MR et al. Selection of highly metastatic variants of different human prostatic carcinomas using orthotopic implantation in nude mice. Clin Cancer Res 1996; 2: 1627-36.

17 Elliott PJ, Ross JS. The proteasome: a new target for novel drug therapies. Am J Clin Pathol 2001; 116: 637-46.

18 Shi J, Tricot G, Szmania S, Rosen N, Garg TK et al. Infusion of haplo-identical killer immunoglobulin-like receptor ligand mismatched NK cells for relapsed myeloma in the setting of autologous stem cell transplantation. Br J Haematol 2008; 143: 641-53. 